\title{
Some inequalities associated with the Hermite-Hadamard-Fejér type for convex function
}

\author{
Mehmet Zeki Sarikaya $\cdot$ Hatice Yaldiz • \\ Samet Erden
}

Received: 27 August 2014/Accepted: 19 December 2014/Published online: 21 January 2015

(C) The Author(s) 2015. This article is published with open access at Springerlink.com

\begin{abstract}
In this paper, we extend some estimates of the right-hand side of a Hermite-Hadamard-Fejér type inequality for functions whose first derivatives' absolute values are convex. The results presented here would provide extensions of those given in earlier works.
\end{abstract}

Keywords Hermite-Hadamard-Fejer inequality ·

Trapezoid inequality · Convex function · Hölder inequality.

Mathematics Subject Classification 26D07 $\cdot 26 \mathrm{D} 15$

\section{Introduction}

Definition 1 The function $f:[a, b] \subset \mathbb{R} \rightarrow \mathbb{R}$ is said to be convex if the following inequality holds

$f(\lambda x+(1-\lambda) y) \leq \lambda f(x)+(1-\lambda) f(y)$

for all $x, y \in[a, b]$ and $\lambda \in[0,1]$. We say that $f$ is concave if $(-f)$ is convex.

The following inequality is well known in the literature as the Hermite-Hadamard integral inequality (see, [2, 4]):

M. Z. Sarikaya $\cdot$ H. Yaldiz $(\square)$

Department of Mathematics, Faculty of Science and Arts, Düzce

University, Konuralp Campus, Düzce, Turkey

e-mail: yaldizhatice@gmail.com

M. Z. Sarikaya

e-mail: sarikayamz@gmail.com

S. Erden

Department of Mathematics, Faculty of Science, Bartın

University, Konuralp Campus, Bartin, Turkey

e-mail: erdem1627@gmail.com $f\left(\frac{a+b}{2}\right) \leq \frac{1}{b-a} \int_{a}^{b} f(x) d x \leq \frac{f(a)+f(b)}{2}$

where $f: I \subset \mathbb{R} \rightarrow \mathbb{R}$ is a convex function on the interval $I$ of real numbers and $a, b \in I$ with $a<b$.

In [1], Dragomir and Agarwal proved the following results connected with the right part of (1.1).

Lemma 1 Let $f: I^{\circ} \subseteq \mathbb{R} \rightarrow \mathbb{R}$ be a differentiable mapping on $I^{\circ}, a, b \in I^{\circ}$ with $a<b$. If $f^{\prime} \in L[a, b]$, then the following equality holds:

$$
\begin{aligned}
& \frac{f(a)+f(b)}{2}-\frac{1}{b-a} \int_{a}^{b} f(x) d x=\frac{b-a}{2} \int_{0}^{1}(1-2 t) f^{\prime} \\
& (t a+(1-t) b) d t .
\end{aligned}
$$

Theorem 1 Let $f: I^{\circ} \subseteq \mathbb{R} \rightarrow \mathbb{R}$ be a differentiable mapping on $I^{\circ}, a, b \in I^{\circ}$ with $a<b$. If $\left|f^{\prime}\right|$ is convex on $[a, b]$, then the following inequality holds:

$$
\left|\frac{f(a)+f(b)}{2}-\frac{1}{b-a} \int_{a}^{b} f(x) d x\right| \leq \frac{(b-a)}{8}\left(\left|f^{\prime}(a)\right|+\left|f^{\prime}(b)\right|\right) \text {. }
$$

Theorem 2 Let $f: I^{\circ} \subset \mathbb{R} \rightarrow \mathbb{R}$ be a differentiable mapping on $I^{\circ}, a, b \in I^{\circ}$ with $a<b, f^{\prime} \in L(a, b)$ and $p>1$. If the mapping $\left|f^{\prime}\right|^{p /(p-1)}$ is convex on $[a, b]$, then the following inequality holds:

$$
\begin{gathered}
\left|\frac{f(a)+f(b)}{2}-\frac{1}{b-a} \int_{a}^{b} f(x) d x\right| \leq \frac{b-a}{2(p+1)^{1 / p}} \\
\times\left(\frac{\left|f^{\prime}(a)\right|^{p /(p-1)}+\left|f^{\prime}(b)\right|^{p /(p-1)}}{2}\right)^{(p-1) / p} .
\end{gathered}
$$

The most well-known inequalities related to the integral mean of a convex function are the Hermite-Hadamard 
inequalities or its weighted versions, the so-called Hermite-Hadamard-Fejér inequalities (see [5-14]). In [3], Fejer gave a weighted generalization of the inequalities (1.1) as the following:

Theorem $3 f:[a, b] \rightarrow \mathbb{R}$ be a convex function, then the inequality

$$
\begin{aligned}
& f\left(\frac{a+b}{2}\right) \int_{a}^{b} w(x) d x \leq \frac{1}{b-a} \int_{a}^{b} f(x) w(x) d x \\
& \quad \leq \frac{f(a)+f(b)}{2} \int_{a}^{b} w(x) d x
\end{aligned}
$$

holds, where $w:[a, b] \rightarrow \mathbb{R}$ is nonnegative, integrable, and symmetric about $x=\frac{a+b}{2}$.

In [5], some inequalities of Hermite-Hadamard-Fejer type for differentiable convex mappings were proved using the following lemma.

Lemma 2 Let $f: I^{\circ} \subset \mathbb{R} \rightarrow \mathbb{R}$ be a differentiable mapping on $I^{\circ}, a, b \in I^{\circ}$ with $a<b$, and $w:[a, b] \rightarrow[0, \infty)$ be a differentiable mapping. If $f^{\prime} \in L[a, b]$, then the following equality holds:

$$
\begin{array}{r}
\frac{f(a)+f(b)}{2} \int_{a}^{b} w(x) d x-\int_{a}^{b} f(x) w(x) d x \\
=\frac{(b-a)^{2}}{2} \int_{0}^{1} p(t) f^{\prime}(t a+(1-t) b) d t
\end{array}
$$

for each $t \in[0,1]$, where

$p(t)=\int_{t}^{1} w(a s+(1-s) b) d s-\int_{0}^{t} w(a s+(1-s) b) d s$.

In this article, using functions whose derivatives' absolute values are convex, we obtained new inequalities of Hermite-Hadamard-Fejer type. The results presented here would provide extensions of those given in earlier works.

\section{Main results}

We will establish some new results connected with the right-hand side of (1.5) and (1.1). Now, we prove our main theorems:

Theorem 4 Let $f: I^{\circ} \subseteq \mathbb{R} \rightarrow \mathbb{R}$ be a differentiable mapping on $I^{\circ}, a, b \in I^{\circ}$ with $a<b$ and let $w:[a, b] \rightarrow \mathbb{R}$ be continuous on $[a, b]$. If $\left|f^{\prime}\right|$ is convex on $[a, b]$, then for all $x \in[a, b]$, the following inequalities hold:

$$
\begin{aligned}
& \left.\mid \int_{x}^{b} w(s) d s\right)^{\alpha} f(b)-\left(\int_{x}^{a} w(s) d s\right)^{\alpha} f(a) \\
& -\alpha \int_{a}^{b}\left(\int_{x}^{t} w(s) d s\right)^{\alpha-1} w(t) f(t) d t \mid \\
& \leq\|w\|_{[a, x], \infty}^{\alpha}\left\{\frac{\left|f^{\prime}(a)\right|}{b-a}\left[\frac{(x-a)^{\alpha+1}(b-x)}{\alpha+1}+\frac{(x-a)^{\alpha+2}}{\alpha+2}\right]\right. \\
& \left.+\frac{\left|f^{\prime}(b)\right|}{b-a} \frac{(x-a)^{\alpha+2}}{(\alpha+1)(\alpha+2)}\right\} \\
& +|| w \|_{[x, b], \infty}^{\alpha}\left\{\frac{\left|f^{\prime}(a)\right|}{b-a} \frac{(b-x)^{\alpha+2}}{(\alpha+1)(\alpha+2)}\right. \\
& \left.+\frac{\left|f^{\prime}(b)\right|}{b-a}\left[\frac{(b-x)^{\alpha+1}(x-a)}{\alpha+1}+\frac{(b-x)^{\alpha+2}}{\alpha+2}\right]\right\} \\
& \leq \frac{\|w\|_{[a, b], \infty}^{\alpha}\left\{| f ^ { \prime } ( a ) | \left[\frac{(x-a)^{\alpha+1}(b-x)}{\alpha+1}\right.\right.}{(b-a)}\left\{\begin{array}{l}
(b-x)^{\alpha+2} \\
\left.\left.+\left|f^{\prime}(b)\right| \mid \frac{(b-x)^{\alpha+1}(x-a)}{\alpha+1}+\frac{(x-a)^{\alpha+2}}{(\alpha+1)(\alpha+2)}+\frac{(b-x)^{\alpha+2}}{\alpha+2}\right]\right\}
\end{array}\right.
\end{aligned}
$$

where $\alpha>0$ and $\|w\|_{\infty}=\sup _{t \in[a, b]}|w(t)|$.

Proof By integration by parts, we have the following equalities:

$$
\begin{aligned}
& \int_{a}^{b}\left(\int_{x}^{t} w(s) d s\right)^{\alpha} f^{\prime}(t) d t \\
& =\left.\left(\int_{x}^{t} w(s) d s\right)^{\alpha} f(t)\right|_{a} ^{b}-\alpha \int_{a}^{b}\left(\int_{x}^{t} w(s) d s\right)^{\alpha-1} \\
& =\left(\int_{x}^{b} w(s) d s\right)^{\alpha} f(b)-\left(\int_{x}^{a} w(s) d s\right)^{\alpha} f(a) \\
& -\alpha \int_{a}^{b}\left(\int_{x}^{t} w(s) d s\right)^{\alpha-1} w(t) f(t) d t .
\end{aligned}
$$$$
=\left.\left(\int_{x}^{t} w(s) d s\right)^{\alpha} f(t)\right|_{a} ^{b}-\alpha \int_{a}^{b}\left(\int_{x}^{t} w(s) d s\right)^{\alpha-1} w(t) f(t) d t
$$

We take absolute value of (2.1) and use convexity of $\left|f^{\prime}\right|$, we find that 


$$
\begin{aligned}
& \mid\left(\int_{x}^{b} w(s) d s\right)^{\alpha} f(b)-\left(\int_{x}^{a} w(s) d s\right)^{\alpha} f(a) \\
& -\alpha \int_{a}^{b}\left(\int_{x}^{t} w(s) d s\right)^{\alpha-1} w(t) f(t) d t \\
& \leq \int_{a}^{x}\left(\left|\int_{x}^{t} w(s) d s\right|\right)^{\alpha}\left|f^{\prime}(t)\right| d t+\int_{x}^{b}\left(\left|\int_{x}^{t} w(s) d s\right|\right)^{\alpha}\left|f^{\prime}(t)\right| d t \\
& \leq\|w\|_{[a, x], \infty}^{\alpha} \int_{a}^{x}(x-t)^{\alpha}\left|f^{\prime}(t)\right| d t+\|w\|_{[x, b], \infty} \int_{x}^{b}(t-x)^{\alpha}\left|f^{\prime}(t)\right| d t \\
& =\|w\|_{[a, x], \infty}^{\alpha}\left[\int_{a}^{x}(x-t)^{\alpha}\left|f^{\prime}\left(\frac{b-t}{b-a} a+\frac{t-a}{b-a} b\right)\right| d t\right] \\
& +\|w\|_{[x, b], \infty}^{\alpha}\left[\int_{x}^{b}(t-x)^{\alpha}\left|f^{\prime}\left(\frac{b-t}{b-a} a+\frac{t-a}{b-a} b\right)\right| d t\right] \\
& \leq\|w\|_{[a, x], \infty}^{\alpha}\left\{\frac{\left|f^{\prime}(a)\right|}{b-a}\left[\frac{(x-a)^{\alpha+1}(b-x)}{\alpha+1}+\frac{(x-a)^{\alpha+2}}{\alpha+2}\right]\right. \\
& \left.+\frac{\left|f^{\prime}(b)\right|}{b-a} \frac{(x-a)^{\alpha+2}}{(\alpha+1)(\alpha+2)}\right\} \\
& +\|w\|_{[x, b], \infty}^{\alpha}\left\{\frac{\left|f^{\prime}(a)\right|}{b-a} \frac{(b-x)^{\alpha+2}}{(\alpha+1)(\alpha+2)}\right. \\
& \left.+\frac{\left|f^{\prime}(b)\right|}{b-a}\left[\frac{(b-x)^{\alpha+1}(x-a)}{\alpha+1}+\frac{(b-x)^{\alpha+2}}{\alpha+2}\right]\right\} \\
& \leq \frac{\|w\|_{[a, b], \infty}^{\alpha}}{(b-a)}\left\{| f ^ { \prime } ( a ) | \left[\frac{(x-a)^{\alpha+1}(b-x)}{\alpha+1}\right.\right. \\
& \left.+\frac{(b-x)^{\alpha+2}}{(\alpha+1)(\alpha+2)}+\frac{(x-a)^{\alpha+2}}{\alpha+2}\right] \\
& \left.+\left|f^{\prime}(b)\right|\left[\frac{(b-x)^{\alpha+1}(x-a)}{\alpha+1}+\frac{(x-a)^{\alpha+2}}{(\alpha+1)(\alpha+2)}+\frac{(b-x)^{\alpha+2}}{\alpha+2}\right]\right\}
\end{aligned}
$$

for all $x \in[a, b]$. Hence, the proof of theorem is completed.

Corollary 1 Under the same assumptions of Theorem 4 with $w(s)=1$, then the following inequality holds:

$$
\begin{aligned}
& \left|(b-x)^{\alpha} f(b)-(a-x)^{\alpha} f(a)-\alpha \int_{a}^{b}(t-x)^{\alpha-1} f(t) d t\right| \\
& \leq \frac{1}{(b-a)}\left\{| f ^ { \prime } ( a ) | \left[\frac{(x-a)^{\alpha+1}(b-x)}{\alpha+1}+\frac{(b-x)^{\alpha+2}}{(\alpha+1)(\alpha+2)}\right.\right. \\
& \left.+\frac{(x-a)^{\alpha+2}}{\alpha+2}\right] \\
& \left.+\left|f^{\prime}(b)\right|\left[\frac{(b-x)^{\alpha+1}(x-a)}{\alpha+1}+\frac{(x-a)^{\alpha+2}}{(\alpha+1)(\alpha+2)}+\frac{(b-x)^{\alpha+2}}{\alpha+2}\right]\right\}
\end{aligned}
$$

for all $x \in[a, b]$.

Remark 1 If we take $\alpha=1$ and $x=\frac{a+b}{2}$ in (2.2), the inequality (2.2) reduces to (1.3).

Corollary 2 (Fejer Type Inequality) Under the same assumptions of Theorem 4 with $\alpha=1$, then the following inequalities hold:

$$
\begin{aligned}
& \left|f(b) \int_{x}^{b} w(s) d s+f(a) \int_{a}^{x} w(s) d s-\int_{a}^{b} w(t) f(t) d t\right| \\
& \leq\left|f^{\prime}(a)\right| \frac{(x-a)^{2}(3 b-2 a-x)\|w\|_{[a, x], \infty}+\|w\|_{[x, b], \infty}(b-x)^{3}}{6(b-a)} \\
& +\left|f^{\prime}(b)\right| \frac{(b-x)^{2}(x-3 a-2 b)\|w\|_{[x, b], \infty}+(x-a)^{3}\|w\|_{[a, x], \infty}}{6(b-a)} \\
& \leq\left|f^{\prime}(a)\right|\left[\frac{(x-a)^{2}(3 b-2 a-x)+(b-x)^{3}}{6(b-a)}\right]\|w\|_{[a, b], \infty} \\
& +\left|f^{\prime}(b)\right|\left[\frac{(b-x)^{2}(x-3 a-2 b)+(x-a)^{3}}{6(b-a)}\right]\|w\|_{[a, b], \infty}
\end{aligned}
$$

which is proved by Tseng et al. in [8].

Corollary 3 (Weighted Trapezoid Inequality) Let $w$ : $[a, b] \rightarrow \mathbb{R}$ be symmetric to $\frac{a+b}{2}$ and $x=\frac{a+b}{2}$ in Corollary 2 . Then the following inequalities hold: 


$$
\begin{aligned}
& \left|\frac{f(a)+f(b)}{2} \int_{a}^{b} w(s) d s-\int_{a}^{b} w(t) f(t) d t\right| \\
& \leq \frac{(b-a)^{2}}{48}\left[5\|w\|_{\left[a, \frac{a+b}{2}\right], \infty}^{\alpha}+\|w\|_{\left[\frac{a+b}{2}, b\right], \infty}^{\alpha}\right]\left|f^{\prime}(a)\right| \\
& \quad+\left[\|w\|_{\left[a, \frac{a+b}{2}\right], \infty}^{\alpha}+5\|w\|_{\left.\left[\frac{a+b}{2}, b\right], \infty\right]}^{\alpha}\right]\left|f^{\prime}(b)\right| \\
& \leq(b-a)^{2}\|w\|_{[a, b], \infty}^{\alpha}\left(\frac{\left|f^{\prime}(a)\right|+\left|f^{\prime}(b)\right|}{8}\right)
\end{aligned}
$$

which is proved by Tseng et al. in [8].

Theorem 5 Let $f: I^{\circ} \subseteq \mathbb{R} \rightarrow \mathbb{R}$ be a differentiable mapping on $I^{\circ}, a, b \in I^{\circ}$ with $a<b$ and let $w:[a, b] \rightarrow \mathbb{R}$ be continuous on $[a, b]$. If $\left|f^{\prime}\right|^{q}$ is convex on $[a, b], q>1$, then for all $x \in[a, b]$, the following inequalities hold:

$$
\begin{aligned}
& \left.\mid \int_{x}^{b} w(s) d s\right)^{\alpha} f(b)-\left(\int_{x}^{a} w(s) d s\right)^{\alpha} f(a) \\
& -\alpha \int_{a}^{b}\left(\int_{x}^{t} w(s) d s\right)^{\alpha-1} w(t) f(t) d t \mid \\
& \leq \frac{(x-a)^{\alpha+\frac{1}{p}}\|w\|_{[a, x], \infty}^{\alpha}}{(b-a)^{\frac{1}{q}}(\alpha p+1)^{\frac{1}{p}}}\left(\frac{(b-a)^{2}-(b-x)^{2}}{2}\left|f^{\prime}(a)\right|^{q}+\frac{(x-a)^{2}}{2}\left|f^{\prime}(b)\right|^{q}\right)^{\frac{1}{q}} \\
& +\frac{(b-x)^{\alpha+\frac{1}{p}}\|w\|_{[x, b], \infty}^{\alpha}}{(b-a)^{\frac{1}{q}}(\alpha p+1)^{\frac{1}{p}}}\left(\frac{(b-x)^{2}}{2}\left|f^{\prime}(a)\right|^{q}+\frac{(b-a)^{2}-(x-a)^{2}}{2}\left|f^{\prime}(b)\right|^{q}\right)^{\frac{1}{q}} \\
& \leq \frac{\|w\|_{[a, b], \infty}^{\alpha}}{(b-a)^{\frac{1}{q}}(\alpha p+1)^{\frac{1}{p}}\left\{(x-a)^{\alpha+\frac{1}{p}}\right.} \\
& \quad \times\left(\frac{(b-a)^{2}-(b-x)^{2}}{2}\left|f^{\prime}(a)\right|^{q}+\frac{(x-a)^{2}}{2}\left|f^{\prime}(b)\right|^{q}\right)^{\frac{1}{q}} \\
& \left.+(b-x)^{\alpha+\frac{1}{p}}\left(\left[\frac{(b-x)^{2}}{2}\left|f^{\prime}(a)\right|^{q}+\frac{(b-a)^{2}-(x-a)^{2}}{2}\left|f^{\prime}(b)\right|^{q}\right]\right)^{\frac{1}{q}}\right\}
\end{aligned}
$$

where $\alpha>0, \frac{1}{p}+\frac{1}{q}=1$, and $\|w\|_{\infty}=\sup _{t \in[a, b]}|w(t)|$.

Proof We take absolute value of (2.1). Using Holder's inequality, we find that

$$
\begin{aligned}
& \mid\left(\int_{x}^{b} w(s) d s\right)^{\alpha} f(b)-\left(\int_{x}^{a} w(s) d s\right)^{\alpha} f(a) \\
& -\alpha \int_{a}^{\alpha-1}\left(\int_{x}^{t} w(s) d s\right)^{\alpha} w(t) f(t) d t \mid \\
& \leq \int_{a}^{x}\left(\left|\int_{x}^{t} w(s) d s\right|\right)^{\prime} f^{\prime}(t) d t+\int_{x}^{b}\left(\left|\int_{x}^{t} w(s) d s\right|\right)^{\alpha} f^{\prime}(t) d t \\
& \leq\left(\int_{a}^{x}\left|\int_{x}^{t} w(s) d s\right|^{\alpha p} d t\right)^{\frac{1}{p}}\left(\int_{a}^{x}\left|f^{\prime}(t)\right|^{q} d t\right)^{\frac{1}{q}} \\
& +\left(\int_{x}^{b}\left|\int_{x}^{t} w(s) d s\right|^{\alpha p} d t\right)^{\frac{1}{p}}\left(\int_{x}^{b}\left|f^{\prime}(t)\right|^{q} d t\right)^{\frac{1}{q}} .
\end{aligned}
$$

Since $\left|f^{\prime}(t)\right|^{q}$ is convex on $[a, b]$

$$
\left|f^{\prime}\left(\frac{b-t}{b-a} a+\frac{t-a}{b-a} b\right)\right|^{q} \leq \frac{b-t}{b-a}\left|f^{\prime}(a)\right|^{q}+\frac{t-a}{b-a}\left|f^{\prime}(b)\right|^{q} .
$$

From (2.3), it follows that

$$
\begin{aligned}
& \mid\left(\int_{x}^{b} w(s) d s\right)^{\alpha} f(b)-\left(\int_{x}^{a} w(s) d s\right)^{\alpha} f(a) \\
& -\alpha \int_{a}^{b}\left(\int_{x}^{t} w(s) d s\right)^{\alpha-1} w(t) f(t) d t \\
& \leq\|w\|_{[a, x], \infty}^{\alpha}\left(\int_{a}^{x}(x-t)^{\alpha p} d t\right)^{\frac{1}{p}} \\
& \times\left(\int_{a}^{x}\left[\frac{b-t}{b-a}\left|f^{\prime}(a)\right|^{q}+\frac{t-a}{b-a}\left|f^{\prime}(b)\right|^{q}\right] d t\right)^{\frac{1}{q}} \\
& +\|w\|_{[x, b], \infty}^{\alpha} \\
& \times\left(\int_{x}^{b}(t-x)^{\alpha p} d t\right)^{\frac{1}{p}}\left(\int_{x}^{b}\left[\frac{b-t}{b-a}\left|f^{\prime}(a)\right|^{q}+\frac{t-a}{b-a}\left|f^{\prime}(b)\right|^{q}\right] d t\right)^{\frac{1}{q}}
\end{aligned}
$$




$$
\begin{aligned}
& \leq \frac{(x-a)^{\alpha+\frac{1}{p}}\|w\|_{[a, x], \infty}^{\alpha}}{(b-a)^{\frac{1}{q}}(\alpha p+1)^{\frac{1}{p}}}\left(\frac{(b-a)^{2}-(b-x)^{2}}{2}\left|f^{\prime}(a)\right|^{q}+\frac{(x-a)^{2}}{2}\left|f^{\prime}(b)\right|^{q}\right)^{\frac{1}{q}} \\
& +\frac{(b-x)^{\alpha+\frac{1}{p}}\|w\|_{[x, b], \infty}^{\alpha}}{(b-a)^{\frac{1}{q}}(\alpha p+1)^{\frac{1}{p}}}\left(\left[\frac{(b-x)^{2}}{2}\left|f^{\prime}(a)\right|^{q}+\frac{(b-a)^{2}-(x-a)^{2}}{2}\left|f^{\prime}(b)\right|^{q}\right]\right)^{\frac{1}{q}} \\
& \leq \frac{\|w\|_{[a, b], \infty}^{\alpha}}{(b-a)^{\frac{1}{q}}(\alpha p+1)^{\frac{1}{p}}}\left\{( x - a ) ^ { \alpha + \frac { 1 } { p } } \left(\frac{(b-a)^{2}-(b-x)^{2}}{2}\left|f^{\prime}(a)\right|^{q}\right.\right. \\
& \left.+\frac{(x-a)^{2}}{2}\left|f^{\prime}(b)\right|^{q}\right)^{\frac{1}{q}} \\
& \left.+(b-x)^{\alpha+\frac{1}{p}}\left(\left[\frac{(b-x)^{2}}{2}\left|f^{\prime}(a)\right|^{q}+\frac{(b-a)^{2}-(x-a)^{2}}{2}\left|f^{\prime}(b)\right|^{q}\right]\right)^{\frac{1}{q}}\right\}
\end{aligned}
$$

which this completes the proof.

Corollary 4 Under the same assumptions of Theorem 5 with $w(s)=1$, then the following inequalities hold:

$$
\begin{aligned}
& \left|(b-x)^{\alpha} f(b)-(a-x)^{\alpha} f(a)-\alpha \int_{a}^{b}(t-x)^{\alpha-1} f(t) d t\right| \\
& \leq \frac{(x-a)^{\alpha+\frac{1}{p}}}{(b-a)^{\frac{1}{q}}(\alpha p+1)^{\frac{1}{p}}}\left(\frac{(b-a)^{2}-(b-x)^{2}}{2}\left|f^{\prime}(a)\right|^{q}+\frac{(x-a)^{2}}{2}\left|f^{\prime}(b)\right|^{q}\right)^{\frac{1}{q}} \\
& +\frac{(b-x)^{\alpha+\frac{1}{p}}}{(b-a)^{\frac{1}{q}}(\alpha p+1)^{\frac{1}{p}}}\left(\frac{(b-x)^{2}}{2}\left|f^{\prime}(a)\right|^{q}+\frac{(b-a)^{2}-(x-a)^{2}}{2}\left|f^{\prime}(b)\right|^{q}\right)^{\frac{1}{q}} \\
& \leq \frac{1}{(b-a)^{\frac{1}{q}}(\alpha p+1)^{\frac{1}{p}}}\left\{( x - a ) ^ { \alpha + \frac { 1 } { p } } \left(\frac{(b-a)^{2}-(b-x)^{2}}{2}\left|f^{\prime}(a)\right|^{q}\right.\right. \\
& \left.+\frac{(x-a)^{2}}{2}\left|f^{\prime}(b)\right|^{q}\right)^{\frac{1}{q}} \\
& +(b-x)^{\alpha+\frac{1}{p}}\left(\left[\frac{(b-x)^{2}}{2}\left|f^{\prime}(a)\right|^{q}+\frac{(b-a)^{2}-(x-a)^{2}}{2} \mid f^{\prime}\right)^{q}\right\}
\end{aligned}
$$

Corollary 5 Let the conditions of Corollary 4 hold. If we take $\alpha=1$ and $x=\frac{a+b}{2}$ in (2.4), then the following inequality holds:

$$
\begin{aligned}
& \left|\frac{f(a)+f(b)}{2}-\frac{1}{b-a} \int_{a}^{b} f(t) d t\right| \\
& \leq \frac{(b-a)}{4(p+1)^{\frac{1}{p}}}\left[\left(\frac{3\left|f^{\prime}(a)\right|^{q}+\left|f^{\prime}(b)\right|^{q}}{4}\right)^{\frac{1}{q}}+\left(\frac{\left|f^{\prime}(a)\right|^{q}+3\left|f^{\prime}(b)\right|^{q}}{4}\right)^{\frac{1}{q}}\right] .
\end{aligned}
$$

Corollary 6 (Fejer Type Inequality) Under the same assumptions of Theorem 5 with $\alpha=1$, then the following inequalities hold:

$$
\begin{aligned}
& \left|f(b) \int_{x}^{b} w(s) d s+f(a) \int_{a}^{x} w(s) d s-\int_{a}^{b} w(t) f(t) d t\right| \\
& \leq \frac{(x-a)^{1+\frac{1}{p}}\|w\|_{[a, x], \infty}}{(b-a)^{\frac{1}{q}}(p+1)^{\frac{1}{p}}}\left(\frac{(b-a)^{2}-(b-x)^{2}}{2}\left|f^{\prime}(a)\right|^{q}\right. \\
& \left.+\frac{(x-a)^{2}}{2}\left|f^{\prime}(b)\right|^{q}\right)^{\frac{1}{q}}+\frac{(b-x)^{1+\frac{1}{p}}\|w\|_{[x, b], \infty}}{(b-a)^{\frac{1}{q}}(p+1)^{\frac{1}{p}}}\left(\frac{(b-x)^{2}}{2}\left|f^{\prime}(a)\right|^{q}\right. \\
& \left.+\frac{(b-a)^{2}-(x-a)^{2}}{2}\left|f^{\prime}(b)\right|^{q}\right)^{\frac{1}{q}} \leq \frac{\|w\|_{[a, b], \infty}}{(b-a)^{\frac{1}{q}}(p+1)^{\frac{1}{p}}}\left\{(x-a)^{1+\frac{1}{p}}\right. \\
& \quad \times\left(\frac{(b-a)^{2}-(b-x)^{2}}{2}\left|f^{\prime}(a)\right|^{q}+\frac{(x-a)^{2}}{2}\left|f^{\prime}(b)\right|^{q}\right)^{\frac{1}{q}} \\
& \left.+(b-x)^{1+\frac{1}{p}}\left(\left[\frac{(b-x)^{2}}{2}\left|f^{\prime}(a)\right|^{q}+\frac{(b-a)^{2}-(x-a)^{2}}{2}\left|f^{\prime}(b)\right|^{q}\right]\right)^{\frac{1}{q}}\right\} .
\end{aligned}
$$

Corollary 7 (Weighted Trapezoid Inequality) Let $w$ : $[a, b] \rightarrow \mathbb{R}$ be symmetric to $\frac{a+b}{2}$ and $x=\frac{a+b}{2}$ in Corollary 6 . Then the following inequalities hold:

$$
\begin{aligned}
& \left|\frac{f(a)+f(b)}{2} \int_{a}^{b} w(s) d s-\int_{a}^{b} w(t) f(t) d t\right| \\
& \leq \frac{(b-a)^{2}}{4(p+1)^{\frac{1}{p}}}\left[\|w\|_{\left[a, \frac{a+b}{2}\right], \infty}\left(\frac{3\left|f^{\prime}(a)\right|^{q}+\left|f^{\prime}(b)\right|^{q}}{4}\right)^{\frac{1}{q}}\right. \\
& \left.\quad+\|w\|_{\left[\frac{a+b}{2}, b\right], \infty}\left(\frac{\left|f^{\prime}(a)\right|^{q}+3\left|f^{\prime}(b)\right|^{q}}{4}\right)^{\frac{1}{q}}\right] \\
& \left.\leq \frac{(b-a)^{2}\|w\|_{[a, b], \infty}\left[\left(\frac{3\left|f^{\prime}(a)\right|^{q}+\left|f^{\prime}(b)\right|^{q}}{4}\right)^{\frac{1}{q}}\right.}{4(p+1)^{\frac{1}{p}}}\left[\frac{\left|f^{\prime}(a)\right|^{q}+3\left|f^{\prime}(b)\right|^{q}}{4}\right)^{\frac{1}{q}}\right] .
\end{aligned}
$$


Theorem 6 Let $f: I^{\circ} \subseteq \mathbb{R} \rightarrow \mathbb{R}$ be a differentiable mapping on $I^{\circ}, a, b \in I^{\circ}$ with $a<b$ and let $w:[a, b] \rightarrow \mathbb{R}$ be continuous on $[a, b]$. If $\left|f^{\prime}\right|^{q}$ is convex on $[a, b], q>1$, then for all $x \in[a, b]$, the following inequality holds:

$$
\begin{aligned}
& \mid\left(\int_{x}^{b} w(s) d s\right)^{\alpha} f(b)-\left(\int_{x}^{a} w(s) d s\right)^{\alpha} f(a) \\
& \quad-\alpha \int_{a}^{b}\left(\int_{x}^{t} w(s) d s\right)^{\alpha-1} w(t) f(t) d t \mid \\
& \leq \frac{(b-a)^{\frac{1}{q}}\|w\|_{[a, b], \infty}^{\alpha}\left[(x-a)^{\alpha p+1}+(b-x)^{\alpha p+1}\right]^{\frac{1}{p}}}{(\alpha p+1)^{\frac{1}{p}}} \\
& \quad \times\left(\frac{\left|f^{\prime}(a)\right|^{q}+\left|f^{\prime}(b)\right|^{q}}{2}\right)^{\frac{1}{q}}
\end{aligned}
$$

where $\alpha>0, \frac{1}{p}+\frac{1}{q}=1$, and $\|w\|_{\infty}=\sup _{t \in[a, b]}|w(t)|$.

Proof We take absolute value of (2.1). Using Holder's inequality and the convexity of $\left|f^{\prime}\right|^{q}$, we find that

$$
\begin{aligned}
& \mid\left(\int_{x}^{b} w(s) d s\right)^{\alpha} f(b)-\left(\int_{x}^{a} w(s) d s\right)^{\alpha} f(a) \\
& -\alpha \int_{a}^{b}\left(\int_{x}^{t} w(s) d s\right)^{\alpha-1} w(t) f(t) d t \\
& \leq\left(\int_{a}^{b}\left|\int_{x}^{t} w(s) d s\right|^{\alpha p} d t\right)^{\frac{1}{p}}\left(\int_{a}^{b}\left|f^{\prime}(t)\right|^{q} d t\right)^{\frac{1}{q}} \\
& \leq\|w\|_{[a, b], \infty}^{\alpha}\left(\int_{a}^{b}|t-x|^{\alpha p} d t\right)^{\frac{1}{p}}\left(\int _ { a } ^ { b } \left[\frac{b-t}{b-a}\left|f^{\prime}(a)\right|^{q}\right.\right. \\
& \left.\left.+\frac{t-a}{b-a}\left|f^{\prime}(b)\right|^{q}\right] d t\right)^{\frac{1}{q}}=\frac{(b-a)^{\frac{1}{q}}\|w\|_{[a, b], \infty}^{\alpha}}{(\alpha p+1)^{\frac{1}{p}}}\left[(x-a)^{\alpha p+1}\right. \\
& \left.+(b-x)^{\alpha p+1}\right]^{\frac{1}{p}}\left(\frac{\left|f^{\prime}(a)\right|^{q}+\left|f^{\prime}(b)\right|^{q}}{2}\right)^{\frac{1}{q}}
\end{aligned}
$$

which this completes the proof.

Corollary 8 Under the same assumptions of Theorem 6 with $w(s)=1$, then the following inequality holds:

$$
\begin{aligned}
& \left|(b-x)^{\alpha} f(b)-(a-x)^{\alpha} f(a)-\alpha \int_{a}^{b}(t-x)^{\alpha-1} f(t) d t\right| \\
& \leq \frac{(b-a)^{\frac{1}{q}}}{(\alpha p+1)^{\frac{1}{p}}}\left[(x-a)^{\alpha p+1}+(b-x)^{\alpha p+1}\right]^{\frac{1}{p}}\left(\frac{\left|f^{\prime}(a)\right|^{q}+\left|f^{\prime}(b)\right|^{q}}{2}\right)^{\frac{1}{q}} .
\end{aligned}
$$

Remark 2 Let the conditions of Corollary 8 hold. If we take $\alpha=1$ and $x=\frac{a+b}{2}$ in (2.5), then the inequality becomes the inequality (1.4).

Corollary 9 (Fejer Type Inequality) Under the same assumptions of Theorem 6 with $\alpha=1$, then the following inequality holds:

$$
\begin{aligned}
& \left|f(b) \int_{x}^{b} w(s) d s+f(a) \int_{a}^{x} w(s) d s-\int_{a}^{b} w(t) f(t) d t\right| \\
& \leq \frac{(b-a)^{\frac{1}{q}}\|w\|_{[a, b], \infty}}{(p+1)^{\frac{1}{p}}}\left[(x-a)^{p+1}+(b-x)^{p+1}\right]^{\frac{1}{p}} \\
& \quad \times\left(\frac{\left|f^{\prime}(a)\right|^{q}+\left|f^{\prime}(b)\right|^{q}}{2}\right)^{\frac{1}{q}} .
\end{aligned}
$$

Corollary 10 (Weighted Trapezoid Inequality) Let $w$ : $[a, b] \rightarrow \mathbb{R}$ be symmetric to $\frac{a+b}{2}$ and $x=\frac{a+b}{2}$ in Corollary 9 . Then the following inequality holds:

$$
\begin{aligned}
& \left|\frac{f(a)+f(b)}{2} \int_{a}^{b} w(s) d s-\int_{a}^{b} w(t) f(t) d t\right| \\
& \leq \frac{(b-a)^{2}\|w\|_{[a, b], \infty}}{2(p+1)^{\frac{1}{p}}}\left(\frac{\left|f^{\prime}(a)\right|^{q}+\left|f^{\prime}(b)\right|^{q}}{2}\right)^{\frac{1}{q}} .
\end{aligned}
$$

Theorem 7 Let $f: I^{\circ} \subseteq \mathbb{R} \rightarrow \mathbb{R}$ be a differentiable mapping on $I^{\circ}, a, b \in I^{\circ}$ with $a<b$ and let $w:[a, b] \rightarrow \mathbb{R}$ be continuous on $[a, b]$. If $\left|f^{\prime}\right|^{q}$ is convex on $[a, b], q>1$, then for all $x \in[a, b]$, the following inequality holds: 


$$
\begin{aligned}
\mid & \left(\int_{x}^{b} w(s) d s\right)^{\alpha} f(b)-\left(\int_{x}^{a} w(s) d s\right)^{\alpha} f(a) \\
& -\alpha \int_{a}^{b}\left(\int_{x}^{t} w(s) d s\right)^{\alpha-1} w(t) f(t) d t \mid \\
\leq & \frac{\|w\|_{[a, b], \infty}^{\alpha}}{(\alpha+1)(\alpha+2)^{\frac{1}{q}}(b-a)^{\frac{1}{q}}}\left((x-a)^{\alpha+1}+(b-x)^{\alpha+1}\right)^{\frac{1}{p}} \\
\times & \left(\left((\alpha+1)(b-a)(x-a)^{\alpha+1}+(b-x)\right.\right. \\
& {\left.\left[(x-a)^{\alpha+1}+(b-x)^{\alpha+1}\right]\right)\left|f^{\prime}(a)\right|^{q} } \\
+ & \left((\alpha+1)(b-a)(b-x)^{\alpha+1}+(x-a)\right. \\
& {\left.\left.\left[(x-a)^{\alpha+1}+(b-x)^{\alpha+1}\right]\right)\left|f^{\prime}(b)\right|^{q}\right)^{\frac{1}{q}} }
\end{aligned}
$$

where $\alpha>0, \frac{1}{p}+\frac{1}{q}=1$, and $\|w\|_{\infty}=\sup _{t \in[a, b]}|w(t)|$.

Proof We take absolute value of (2.1). Using Holder's inequality and the convexity of $\left|f^{\prime}\right|^{q}$, we find that

$$
\begin{aligned}
& \mid\left(\int_{x}^{b} w(s) d s\right)^{\alpha} f(b)-\left(\int_{x}^{a} w(s) d s\right)^{\alpha} f(a) \\
& -\alpha \int_{a}^{b}\left(\int_{x}^{t} w(s) d s\right)^{\alpha-1} w(t) f(t) d t \\
& \leq\left(\int_{a}^{b}\left|\int_{x}^{t} w(s) d s\right|^{\alpha} d t\right)^{\frac{1}{p}}\left(\left.\left.\int_{a}^{b}\left|\int_{x}^{t} w(s) d s\right|\right|^{\alpha}(t)\right|^{q} d t\right)^{\frac{1}{q}} \\
& \leq\|w\|_{[a, b], \infty}^{\alpha}\left(\int_{a}^{b}|t-x|^{\alpha} d t\right)^{\frac{1}{p}}\left(\int _ { a } ^ { b } | t - x | ^ { \alpha } \left[\frac{b-t}{b-a}\left|f^{\prime}(a)\right|^{q}\right.\right. \\
& \left.\left.+\frac{t-a}{b-a}\left|f^{\prime}(b)\right|^{q}\right] d t\right)^{\frac{1}{q}}=\|w\|_{[a, b], \infty}^{\alpha}\left(\frac{(x-a)^{\alpha+1}+(b-x)^{\alpha+1}}{\alpha+1}\right)^{\frac{1}{p}} \\
& \times\left(\left(\frac{(b-x)(x-a)^{\alpha+1}}{\alpha+1}+\frac{(x-a)^{\alpha+2}}{\alpha+2}\right)\left|f^{\prime}(a)\right|^{q}\right. \\
& +\left(\frac{(x-a)^{\alpha+2}}{(\alpha+1)(\alpha+2)}\right)\left|f^{\prime}(b)\right|^{q}+\left(\frac{(b-x)^{\alpha+2}}{(\alpha+1)(\alpha+2)}\right)\left|f^{\prime}(a)\right|^{q} \\
& \left.+\left(\frac{(x-a)(b-x)^{\alpha+1}}{\alpha+1}+\frac{(b-x)^{\alpha+2}}{\alpha+2}\right)\left|f^{\prime}(b)\right|^{q}\right)^{\frac{1}{q}} \\
& =\frac{\|w\|_{[a, b], \infty}^{\alpha}}{(\alpha+1)(\alpha+2)^{\frac{1}{q}}(b-a)^{\frac{1}{q}}}\left((x-a)^{\alpha+1}+(b-x)^{\alpha+1}\right)^{\frac{1}{p}} \\
& \times\left(\left((\alpha+1)(b-a)(x-a)^{\alpha+1}+(b-x)\right.\right. \\
& \left.\left[(x-a)^{\alpha+1}+(b-x)^{\alpha+1}\right]\right)\left|f^{\prime}(a)\right|^{q} \\
& +\left((\alpha+1)(b-a)(b-x)^{\alpha+1}+(x-a)\right. \\
& \left.\left.\left[(x-a)^{\alpha+1}+(b-x)^{\alpha+1}\right]\right)\left|f^{\prime}(b)\right|^{q}\right)^{\frac{1}{q}}
\end{aligned}
$$

which this completes the proof.

Corollary 11 Under the same assumptions of Theorem 7 with $w(s)=1$, then the following inequality holds:

$$
\begin{aligned}
\mid & (b-x)^{\alpha} f(b)-(a-x)^{\alpha} f(a)-\alpha \int_{a}^{b}(t-x)^{\alpha-1} f(t) d t \mid \\
\leq & \frac{1}{(\alpha+1)(\alpha+2)^{\frac{1}{q}}(b-a)^{\frac{1}{q}}}\left((x-a)^{\alpha+1}+(b-x)^{\alpha+1}\right)^{\frac{1}{p}} \\
\times & \left(\left((\alpha+1)(b-a)(x-a)^{\alpha+1}+(b-x)\right.\right. \\
+ & \left((\alpha+1)(b-a)(b-x)^{\alpha+1}+(x-a)\right. \\
& {\left.\left.\left[(x-a)^{\alpha+1}+(b-x)^{\alpha+1}\right]\right)\left|f^{\prime}(b)\right|^{q}\right)^{\frac{1}{q}} . }
\end{aligned}
$$

Corollary 12 Let the conditions of Corollary 11 hold. If we take $\alpha=1$ and $x=\frac{a+b}{2}$ in (2.6), then the following inequality holds:

$$
\begin{aligned}
& \left|\frac{f(a)+f(b)}{2}-\frac{1}{b-a} \int_{a}^{b} f(t) d t\right| \\
& \quad \leq \frac{(b-a)}{4}\left(\frac{\left|f^{\prime}(a)\right|^{q}+\left|f^{\prime}(b)\right|^{q}}{2}\right)^{\frac{1}{q}} .
\end{aligned}
$$

Corollary 13 (Fejer Type Inequality) Under the same assumptions of Theorem 7 with $\alpha=1$, then the following inequality holds:

$$
\begin{aligned}
& \left|f(b) \int_{x}^{b} w(s) d s+f(a) \int_{a}^{x} w(s) d s-\int_{a}^{b} w(t) f(t) d t\right| \\
& \leq \frac{\|w\|_{[a, b], \infty}^{\alpha}}{2 \cdot 3^{\frac{1}{q}}(b-a)^{\frac{1}{q}}}\left((x-a)^{2}+(b-x)^{2}\right)^{\frac{1}{p}} \\
& \quad \times\left(\left(2(b-a)(x-a)^{2}+(b-x)\left[(x-a)^{2}+(b-x)^{2}\right]\right)\left|f^{\prime}(a)\right|^{q}\right. \\
& \left.\quad+\left(2(b-a)(b-x)^{2}+(x-a)\left[(x-a)^{2}+(b-x)^{2}\right]\right)\left|f^{\prime}(b)\right|^{q}\right)^{\frac{1}{q}} .
\end{aligned}
$$

Corollary 14 (Weighted Trapezoid Inequality) Let $w$ : $[a, b] \rightarrow \mathbb{R}$ be symmetric to $\frac{a+b}{2}$ and $x=\frac{a+b}{2}$ in Corollary 13. Then the following inequality holds:

$$
\begin{aligned}
& \left|\frac{f(a)+f(b)}{2} \int_{a}^{b} w(s) d s-\int_{a}^{b} w(t) f(t) d t\right| \\
& \leq \frac{(b-a)^{2}\|w\|_{[a, b], \infty}^{\alpha}}{4}\left(\frac{\left|f^{\prime}(a)\right|^{q}+\left|f^{\prime}(b)\right|^{q}}{2}\right)^{\frac{1}{q}} .
\end{aligned}
$$


Open Access This article is distributed under the terms of the Creative Commons Attribution License which permits any use, distribution, and reproduction in any medium, provided the original author(s) and the source are credited.

\section{References}

1. Dragomir, S.S., Agarwal, R.P.: Two inequalities for differentiable mappings and applications to special means of real numbers and to trapezoidal formula. Appl. Math. Lett. 11(5), 91-95 (1998)

2. Dragomir, S.S., Pearce, C.E.M.: Selected topics on HermiteHadamard inequalities and applications. Victoria University, RGMIA Monographs (2000)

3. Fejer, L.: Über die Fourierreihen, II. Math. Naturwiss. Anz Ungar. Akad. Wiss. (Hungarian) 24, 369-390 (1906)

4. Pečarić, J., Proschan, F., Tong, Y.L.: Convex functions, partial ordering and statistical applications. Academic Press, New York (1991)

5. Sarikaya, M.Z.: On new Hermite Hadamard Fejer type integral inequalities. Stud. Universit. Babes Bolyai Math. 57(3), 377-386 (2012)

6. Sarikaya, M.Z., Erden, S.: On the weighted integral inequalities for convex function. RGMIA research report collection. 17(70), $10(2014)$
7. Sarikaya, M.Z., Erden, S.: On the Hermite-Hadamard-Fejér type integral inequality for convex function. RGMIA research report collection. 17(69), 12 (2014)

8. Tseng, K.-L., Yang, G.-S., Hsu, K.-C.: Some inequalities for differentiable mappings and applications to Fejer inequality and weighted trapezoidal formula. Taiwan. J. Math. 15(4), 1737-1747 (2011)

9. Hwang, S.-R., Tseng, K.-L., Hsu, K.-C.: Hermite-Hadamard type and Fejér type inequalities for general weights (I). J. Inequal. Appl. 2013, 170 (2013)

10. Wang, C.-L., Wang, X.-H.: On an extension of Hadamard inequality for convex functions. Chin. Ann. Math. 3, 567-570 (1982)

11. Wasowicz, S., Witkonski, A.: On some inequality of HermiteHadamard type. Opuscu. Math. 32(2), 591-600 (2012)

12. $\mathrm{Wu}, \mathrm{S} .-\mathrm{H} .:$ On the weighted generalization of the HermiteHadamard inequality and its applications. Rocky Mt. J. Math. 39(5), 1741-1749 (2009)

13. Xi, B.-Y., Qi, F.: Some Hermite-Hadamard type inequalities for differentiable convex functions and applications. Hacet. J. Math. Stat. 42(3), 243-257 (2013)

14. Xi, B.-Y., Qi, F., Hermite-Hadamard type inequalities for functions whose derivatives are of convexities. Nonlinear Funct. Anal. Appl. 18(2), 163-176 (2013) 